\title{
Mismatch Negativity contribution in Multiple Sclerosis patients
}

\author{
Marco Aurélio Rocha Santos ${ }^{1}$, Mário Sérgio Lei \\ Munboz ${ }^{2}$, Marco Aurélio L. Peixoto ${ }^{3}$, Vitor Geraldi \\ Haase ${ }^{4}$,Jussara L. Rodrigues, Luciana Macedo \\ Resende $^{6}$
}

Keywords: attention, auditory cortex, multiple sclerosis, evoked potentials.

\section{Summary}

\begin{abstract}
$\mathrm{M}$ ismatch Negativity is a functional index of the supratemporal auditory cortex. Aim: The aim of the present study on Mismatch Negativity (MMN) in multiple sclerosis (MS) patients was to evaluate if the MMN can be correlated with cognitive deficits assessed by the Paced Auditory Addition Task - PASAT. Method: a clinical study in forty females and 20 males separated in two groups: control individuals and those with a definite diagnosis of MS underwent a duration and frequncy MMN. The MMN latencies and negative amplitudes obtained from the MS group were compared to the ones from the control group. The scores from the Paced Auditory Addition Task were correlated either with the presence or the absence of MMN. Results: MMN was found in $60 \%$ of the individuals with multiple sclerosis within the auditory stimulation protocol with varied durations, and in $45 \%$ within the auditory stimulation protocol with frequency variations. There were no statistically significant differences in latencies and amplitudes when compared to controls. We found a statistically significant correlation for the lack of MMN wave together with cognitive disorder asserted by the PASAT. Conclusions: The MMN correlated to the cognitive deficit assessed by the PASAT.
\end{abstract}

${ }^{1} \mathrm{PhD}$ in Sciences, Postgraduate Program in Otorhinolaryngology and Head and Neck Surgery - Federal University of São Paulo - UNIFESP - Paulista School of Medicine - Otorhinolaryngologist.

${ }^{2}$ Associate Professor - Federal University of São Paulo, UNIFESP.

${ }^{3} \mathrm{PhD}$ in Neurology, Assistant Professor of Ophthalmology and Neurology - Medical School - UFMG.

${ }^{4} \mathrm{PhD}$ in Medical Psychology, Neurologist, Assistant Professor - Department of Psychology - UFMG.

${ }^{5}$ M.S student in Developmental Psychology - UFMG, Psychologist.

${ }^{6}$ MS in Speech and Hearing Therapy - Pontifícia Universidade Católica de São Paulo, Assistant Professor of Speech and Hearing Therapy - Universidade Federal de Minas Gerais.

Discipline of Otoneurology - Federal University of São Paulo UNIFESP - Paulista School of Medicine - Multiple Sclerosis Investigation Center of Minas Gerais - CIEM-MG - Medical School - Federal University of Minas Gerais

Mailing Address: Rua dos Otoni 927/501 Bairro Santa Efigênia Belo Horizonte Minas Gerais 30150-270

Telefax: (0xx31) 3222-1019/32747375 - E-mail: marcoarsantos@uol.com.br

Paper submitted to the ABORL-CCF SGP (Management Publications System) on February 11th, 2006, and approved on August 7th, 2006. cod. 1720 


\section{INTRODUCTION}

Evoked Potentials (EPs) are brain electrical manifestations in response to external stimuli. The clinical usefulness of these potentials is based on the capacity to: show abnormal function of the sensorial system; reveal dysfunctions of the sensorial system in demyelination diseases; aid in the definition of disease anatomical distribution and objectively monitor changes in the clinical conditions of patients1. EPs are small characteristic variations in the electroencephalogram, related to sensorial, motor or cognitive stimuli, thus becoming a neurophysiological indicator ${ }^{1,2}$.

EPs may be classified as sensorial or exogenous and endogenous or cognitive ${ }^{1,2}$. Exogenous potentials are determined by the physical and temporal characteristics of the stimuli and are directly related to them. The endogenous depend on the individual, the task performed, and the stimuli content and are correlated to brain processing activities $^{3}$.

Recent progress in brain functioning and the development of anatomical brain activity localization in human beings who perform specific tasks, has allowed for these new clinical applications for brain $\mathrm{EPs}^{4}$.

One of the EPs components, called Mismatch Negativity (MMN)5 allowed the development of objective procedures for physiological assessments of great interest to neurology, psychiatry and otorhinolaryngology $y^{4}$.

MMN is one endogenous component of EPs, obtained when we present to a subject or patient one block of hundreds of identical stimuli (patterns) which are randomly replaced by acoustically different stimuli (deviants). These stimuli, patterns and deviants should bear mild differences in acoustic characteristics, such as: duration, frequency, intensity, spatial location or, still, by the use of phonemes or music notes. In order to obtain MMN, it is necessary that acoustic stimuli attention be distracted by a visual task.

We obtain the MMN subtracting responses elicited by the standard stimuli of deviant stimuli responses, recording a negative wave that, depending on the deviant stimuli amplitude appears between 100 and 250 milliseconds (ms) after the stimuli onset.

The negativity is generated by changes in the discrimination process that occur in the auditory cortex and reflects the auditory sensorial memory. It may be used as an indicator of the cortex functional status, of perceptive sensorial capacity, as an index of the hearing processing and the neural plasticity of the auditory system ${ }^{6}$.

$\mathrm{MMN}$ is a reproducible potential7, triggered when there is a difference between standard stimuli and the frequency deviants in duration, in intensity, in spatial location, in phonetic characteristics, rhythmic characteristics and temporal pattern. These differences among stimuli should not be large ${ }^{8}$, and it may alter both the latency and the amplitude of stimuli duration ${ }^{11}$ and intensity ${ }^{12}$ may interfere in the establishment of neural representation, and should be properly controlled.

Multiple Sclerosis (MS) is an immune-mediated, demyelinating, inflammatory disease that affects the nervous system. It is mainly found in young female adults, presenting periods of specific symptoms worsening and remission. Alterations are frequent in the optic nerve, the spinal chord, and the brain stem and periventricular regions. Diagnosis is clinical, based on spatial and temporal lesion spread to the Central Nervous System. MS diagnosis is clinically defined by the Poser criteria - the occurrence of at least two spells and clinical evidence of two separate lesions, or two spells and clinical evidence of one lesion and paraclinical evidence of another. These two spells should involve different parts of the Central Nervous System, being one month apart, and each one lasting for at least 24 hours $^{13}$. Complementary tests that support clinical diagnosis are: MRI, CSF exam to search for oligoclonal bands, IgG formation rate in the CSF; visual, somatosensorial and auditory evoked potentials.

MS patient assessment by means of long latency auditory evoked potentials ${ }^{14}$ has shown alterations in wave latency and shape ${ }^{15,16}$, as its correlation with altered neuropsychological tests ${ }^{17}$.

Cognitive alterations may affect MS patients. Neuropsychological studies show that cognitive dysfunction happens in 54 to $65 \%{ }^{18}$ of patients and these may alter memory, attention, verbal fluency, task performance and visual perception19. The Compound Functional Measure for Multiple Sclerosis (MSFC)20 was developed based on psychometric tests in patients with MS, bearing the following tests: 25 feet timed walk; 9 pins in the hole test, and the Paced Auditory Addition Test - PASAT ${ }^{21}$.

Neurological tests are very useful in assessing cognitive function, among them, the PASAT ${ }^{21}$ is a sensitive and practical test used to assess MS cognitive involvement. The PASAT scores of each MS patient are compared to a control group that matches the former in age, educational level and socio-economical status ${ }^{22}$.

\section{OBJECTIVES}

The goals of the present study are: to use MMN in clinically diagnosed MS patients and investigate whether or not MMN can be used as a functional index of the supratemporal auditory cortex and analyze if MMN alterations correlate to the cognitive impairment assessed by the PASAT test.

\section{MATERIALS AND METHODS}

The Research Ethics Committee of the Federal University of São Paulo/São Paulo Hospital analysed and approved the project, protocol \#0 0638/03. 
40 females and 20 males participated on this study. They were further divided in 2 groups: Control group and MS group, who read and signed an informed consent approved by the Research Ethics Committee.

For the MS group, we selected both men and women: 30 women with ages varying between 26 and 58 years, 43.1 years of average and 10 men, between 29 and 57 years - 39.4 of mean age. All the patients in this latter group had Clinically Established diagnosis of Multiple Sclerosis, according to Poser criteria, and did not have signs of MS worsening at the time of the tests and the MMN recording, or visual disorders that could interfere in the visual distraction task. All patients underwent an otorhinolaryngology exam and tonal audiometry in order to assess tonal thresholds at the frequencies of 500, 1,000 and 2,000 $\mathrm{Hz}$.

To make up the control group, we selected individuals of both genders, with ages matching those from the MS group: 10 women with ages varying between 27 and 50 years, mean age of 40.1 years; and 10 men, between 25 and 55 years, mean age of 37.9, without prior history of neurologic or otorhinolaryngological disorders. They all underwent otorhinolaryngological exam, not showing alterations at the otoscopy and tonal audiometry thresholds below or equal to $25 \mathrm{~dB} \mathrm{HL}$ at the frequencies of 500 , 1,000 and 2,000 Hz. None of them used drugs that could affect the Central Nervous System.

For MMN recording, we used a group of stimuli with duration variation and another group with frequency variation, presented by means of TDH-39 supra-aural phones.

In the stimuli group that varied the duration, we used tones at a $55 \mathrm{~dB}$ HL intensity, in both ears, with a total of 1,000 tones, $80 \%$ of standard stimuli and $20 \%$ deviant stimuli, at a frequency of $750 \mathrm{~Hz}$, the standard stimuli lasted for $75 \mathrm{~ms}$ and the deviants lasted $25 \mathrm{~ms}$. Deviant tones were randomly assigned by the computer among the standard ones. As stimuli rate, we used 1.1 stimulus per second $(/ \mathrm{s})$. The standard tones used had linear ascending and descending times, plateauing in $55 \mathrm{~ms}$. Deviant tones had $5 \mathrm{~ms}$ of linear ascending and descending times, with a $15 \mathrm{~ms}$ plateau.

In the frequency variation stimulus group, we used tones at $55 \mathrm{~dB} \mathrm{HL}$, on both ears, at a total of 1,000 tones, $80 \%$ of standard stimuli and $20 \%$ deviant stimuli, lasting for $50 \mathrm{~ms}$; standard stimuli at a frequency of $1,000 \mathrm{~Hz}$ and deviant stimuli at a frequency of 1,500 Hz. Deviant tones were randomly assigned by the computer among the standard tones. As stimulus rate, we used 1.1 stimulus /s. Both standard and deviant tones had $10 \mathrm{~ms}$ of ascending and descending linear time, with $30 \mathrm{~ms}$ plateau, both tones with linear ramp.

Recordings were carried out in a sound-treated environment, with the subjects comfortably laying on their backs, with a $45^{\circ}$ upward head tilt and the head was supported by a pillow in order to relax neck muscles, and as a distraction visual task we showed them a subtitled movie, without sound, on a video screen. All recordings were carried out at the same time, in the morning.

For this investigation we used a two-channel Biologic ${ }^{\circledR}$ evoked potentials equipment, and the recording was carried out by silver electrodes placed on the midline of the forehead and central, in the Fz and $\mathrm{Cz}$ leads, the ear lobes were used as reference points, and a lead on the nose tip was used as ground wire, they were attached by means of an adhesive tape, after skin raking and electrolytic paste was used on the skin. Electrodes impedances were kept bellow $5 \mathrm{~K}$ ohms.

We used $512 \mathrm{~ms}$ as analysis time, high pass filters at $0.1 \mathrm{~Hz}$ and low pass at $100 \mathrm{~Hz}$.

Signal acquisition onset was adjusted with the exact onset of hearing stimulation.

MMN was considered as maximum negative peak between 100 and $250 \mathrm{~ms}$, in $\mathrm{Fz} \mathrm{e} \mathrm{Cz}$, obtained by the difference curve, subtracting the standard stimulus response curve from the deviant stimuli response curves. We analyzed difference wave shapes, latencies and negative amplitude measured in ms e $\mu \mathrm{V}$. We also analyzed MMN presence or absence after wave subtraction.

The group with MS underwent MSFC test, and the Paced Auditory Addition Test - PASAT was used as a cognitive impairment index. PASAT was carried out in the version with 3 seconds inter-stimuli intervals which were numbered from 1 to 9 in pseudo-randomized sequence, stored in a Compact Disk. The subject's task was to add up the numbers two by two and provide the result, as they heard the next number, the third, add it to the previous one, according to the Brazilian Portuguese version adapted by Haase et al.14. The PASAT raw score was defined by the number of correct answers for the summations.

Disorders seen at the PASAT were defined as a raw score equal to or below 27. Above this value. The PASAT was considered normal.

MMN presence or absence, in both auditory stimulation protocols, was compared to the presence of cognitive impairment assessed by the PASAT.

The age means were compared by the Variance Analysis Test (ANOVA) between genders and MS and control groups.

The ANOVA test was applied in order to compare MMN negative amplitude and latencies between the control and MS groups, between the genders and the recording leads ( $\mathrm{Fz}$ and $\mathrm{Cz}$ ).

We used the Fischer test in order to compare MMN presence or absence with cognitive impairment assessed by PASAT.

For both tests we used 5\% as significance level. 
Table 1. Statistical analysis and comparison of the mean ages and standard deviations between genders and the groups.

\begin{tabular}{cccc}
\hline Group & Statistics & Females & Males \\
\hline \multirow{2}{*}{$\mathrm{C}$} & Mean & 40,10 & 37,90 \\
& Standard Deviation & 8,319 & 10,826 \\
& $\mathrm{n}$ & 10 & 10 \\
\multirow{2}{*}{ MS } & Mean & 43,10 & 39,40 \\
& Standard Deviation & 8,130 & 10,298 \\
& $\mathrm{n}$ & 30 & 10 \\
\hline
\end{tabular}

C - Control Group

MS - Group with Multiple Sclerosis

ANOVA

\begin{tabular}{cc}
\hline Effect & $F(p)$ Significance \\
\hline Group & 0,3908 \\
Gender & 0,2617 \\
Group x Gender & 0,7742 \\
\hline
\end{tabular}

\section{RESULTS}

Table 1 shows the statistical analysis and comparisons of age means between genders and the groups.

MMN was recorded in 60\% of the patients in the duration variation protocol, and in $45 \%$ in the group with frequency variation protocol in the group of patients

Table 2. MMN latency standard deviation and mean in the hearing stimulation protocol with duration variation in males and females, in the $\mathrm{Cz}$ and $\mathrm{Fz}$ leads, in both controls and MS patients.

\begin{tabular}{cccccc}
\hline & & \multicolumn{2}{c}{ Females } & \multicolumn{2}{c}{ Males } \\
\hline Group & Statistics & $\mathrm{Cz}$ & $\mathrm{Fz}$ & $\mathrm{Cz}$ & $\mathrm{Fz}$ \\
\hline \multirow{2}{*}{$\mathrm{C}$} & Mean & 194,60 & 192,40 & 180,20 & 178,80 \\
& Standard Deviation & 30,51 & 27,79 & 24,63 & 25,13 \\
& $\mathrm{n}$ & 10 & 10 & 10 & 10 \\
\hline \multirow{2}{*}{ MS } & Mean & 180,44 & 182,11 & 183,20 & 172,40 \\
& Standard Deviation & 26,59 & 29,64 & 41,00 & 44,05 \\
& $\mathrm{n}$ & 18 & 18 & 5 & 6 \\
\hline
\end{tabular}

C - Control Group

MS - Group with Multiple Sclerosis

with MS. The latter did not show statistically significant differences in MMN negative amplitude and latencies between the MS and control groups in both genders and leads (Tables 2 to 5).

Tables 6 and 7 show the statistical analysis and comparisons between MMN presence or absence and cognitive
Table 3. MMN negative amplitude mean and standard deviation in the hearing stimulation protocol with duration variation for males and females, in the $\mathrm{Cz}$ and $\mathrm{Fz}$ leads in both Control and MS groups.

\begin{tabular}{cccccc}
\hline & & \multicolumn{2}{c}{ Females } & \multicolumn{2}{c}{ Males } \\
\hline \multirow{2}{*}{ Group } & Statistics & $\mathrm{Cz}$ & $\mathrm{Fz}$ & $\mathrm{Cz}$ & $\mathrm{Fz}$ \\
& Mean & $-3,22$ & $-3,66$ & $-3,23$ & $-3,48$ \\
$\mathrm{C}$ & Standard Deviation & 1,51 & 1,70 & 0,83 & 1,33 \\
& $\mathrm{n}$ & 10 & 10 & 10 & 10 \\
\hline \multirow{4}{*}{ MS } & Mean & $-2,76$ & $-2,82$ & $-2,87$ & $-2,81$ \\
& Standard Deviation & 1,34 & 0,93 & 0,90 & 0,85 \\
C - Control Group & 18 & 18 & 5 & 6 \\
MS - Group with Multiple Sclerosis & & & &
\end{tabular}

Table 4. MMN latency mean and standard deviation in the hearing stimulation protocol with frequency variation for males and females, in the $\mathrm{Cz}$ and $\mathrm{Fz}$ leads in both Control and MS groups.

\begin{tabular}{cccccc}
\hline & & \multicolumn{2}{c}{ Females } & \multicolumn{2}{c}{ Males } \\
\hline \multirow{2}{*}{ Group } & Statistics & Cz & Fz & Cz & Fz \\
& Mean & 148,20 & 147,60 & 158,40 & 160,80 \\
C & Standard Deviation & 41,58 & 42,27 & 37,65 & 36,83 \\
& $\mathrm{n}$ & 10 & 10 & 10 & 10 \\
\hline \multirow{2}{*}{ MS } & Mean & 153,23 & 152,62 & 181,20 & 180,00 \\
& Standard Deviation & 43,35 & 41,69 & 31,13 & 34,09 \\
& $\mathrm{n}$ & 13 & 13 & 5 & 5 \\
\hline
\end{tabular}

C - Control Group

EM - Multiple Sclerosis Group

Table 5. MMN negative amplitude mean and standard deviation in the hearing stimulation protocol with frequency variation for males and females, in the $\mathrm{Cz}$ and $\mathrm{Fz}$ leads in both Control and MS groups.

\begin{tabular}{cccccc}
\hline & & \multicolumn{2}{c}{ Females } & \multicolumn{2}{c}{ Males } \\
\hline Group & Statistics & $\mathrm{Cz}$ & $\mathrm{Fz}$ & $\mathrm{Cz}$ & $\mathrm{Fz}$ \\
\hline \multirow{2}{*}{$\mathrm{C}$} & Mean & $-1,77$ & $-2,32$ & $-2,62$ & $-1,89$ \\
& Standard Deviation & 2,13 & 1,02 & 1,37 & 2,15 \\
& $\mathrm{n}$ & 10 & 10 & 10 & 10 \\
\hline \multirow{4}{*}{$\mathrm{MS}$} & Mean & $-2,29$ & $-2,18$ & $-2,70$ & $-2,15$ \\
& Standard Deviation & 0,97 & 1,28 & 1,51 & 1,86 \\
C - Control Group & 13 & 13 & 5 & 5 \\
EM - Multiple Sclerosis Group & & & &
\end{tabular}


Table 6. Statistical analysis and comparison between MMN presence or absense in the hearing stimulation protocol with duration variation and the presence of impairment, assessed by PASAT in the MS group.

\begin{tabular}{ccccccccc}
\hline & \multicolumn{3}{c}{ Duration } & \multicolumn{4}{c}{ Test } \\
\hline & Absent & \multicolumn{2}{c}{ Present } & \multicolumn{2}{c}{ Total } & Fisher \\
\hline PASAT & $\mathrm{n}$ & $\%$ & $\mathrm{n}$ & $\%$ & $\mathrm{n}$ & $\%$ & $(\mathrm{p})$ \\
\hline $\begin{array}{c}\text { Without impair- } \\
\text { ment }\end{array}$ & 2 & 12,5 & 15 & 62,5 & 17 & 42,5 & \\
$\begin{array}{c}\text { With impairment } \\
\text { Total }\end{array}$ & 14 & 87,5 & 9 & 37,5 & 23 & 57,5 & $0,003^{*}$ \\
& 16 & 100,0 & 24 & 100,0 & 40 & 100,0 & \\
\hline
\end{tabular}

Table 7. Statistical analysis and comparison between MMN presence or absence in the hearing stimulation protocol with frequency variation and the presence of impairment, assessed by PASAT in the MS group.

\begin{tabular}{ccccccccc}
\hline & \multicolumn{3}{c}{ Duration } & \multicolumn{4}{c}{ Test } \\
\hline & Absent & \multicolumn{1}{c}{ Present } & \multicolumn{2}{c}{ Total } & Fisher \\
\hline PASAT & $\mathrm{n}$ & $\%$ & $\mathrm{n}$ & $\%$ & $\mathrm{n}$ & $\%$ & $(\mathrm{p})$ \\
\hline $\begin{array}{c}\text { Without impair- } \\
\text { ment }\end{array}$ & 5 & 21,73 & 12 & 66,6 & 17 & 42,5 & \\
$\begin{array}{c}\text { With impairment } \\
\text { Total }\end{array}$ & 17 & 73,91 & 6 & 33,3 & 23 & 57,5 & $0,010^{*}$ \\
& 23 & 100,0 & 18 & 100,0 & 40 & 100,0 & \\
\hline
\end{tabular}

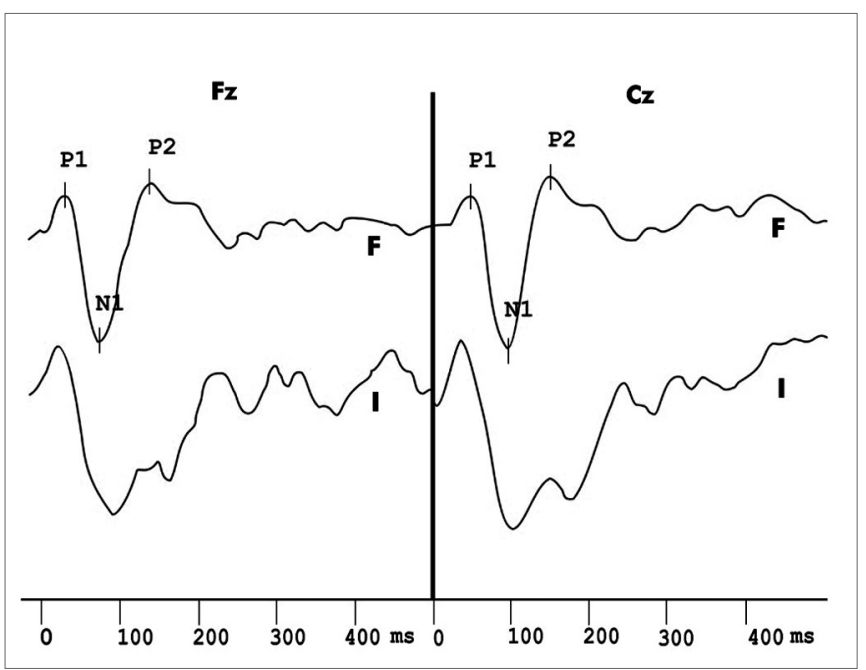

Figure 1. Exogenous long latency hearing evoked potentials recording in the duration variation protocol in the control group. $\mathrm{F}$ - recording in response to frequent stimulus I - recording in response to infrequent stimuli

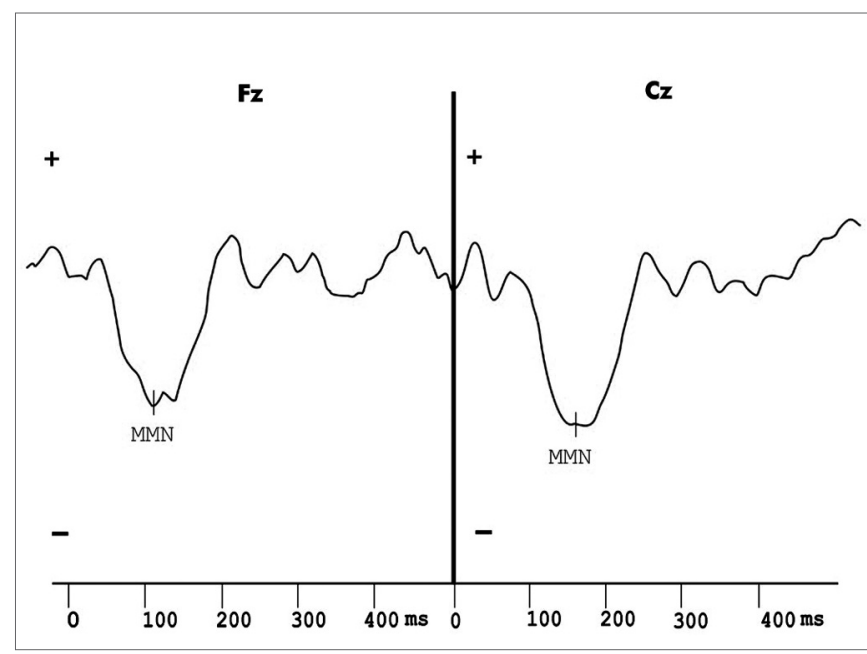

Figure 2. Mismatch Negativity shown by the difference wave obtained with frequent and infrequent stimuli in the hearing stimulation protoco with duration variation in the control group.

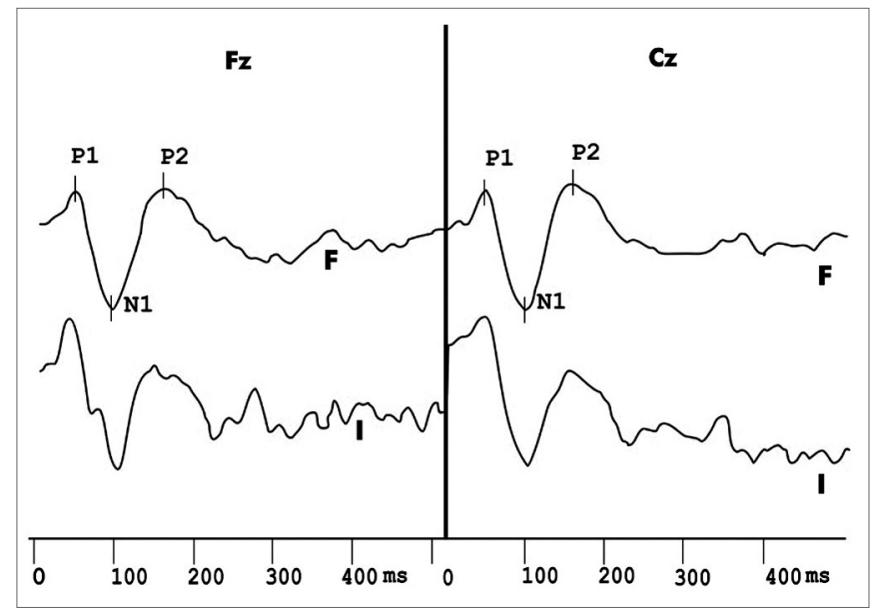

Figure 3. Exogenous long latency hearing evoked potentials with duration variation in the MS group. F - Frequent stimuli response record I - infrequent stimuli response record 


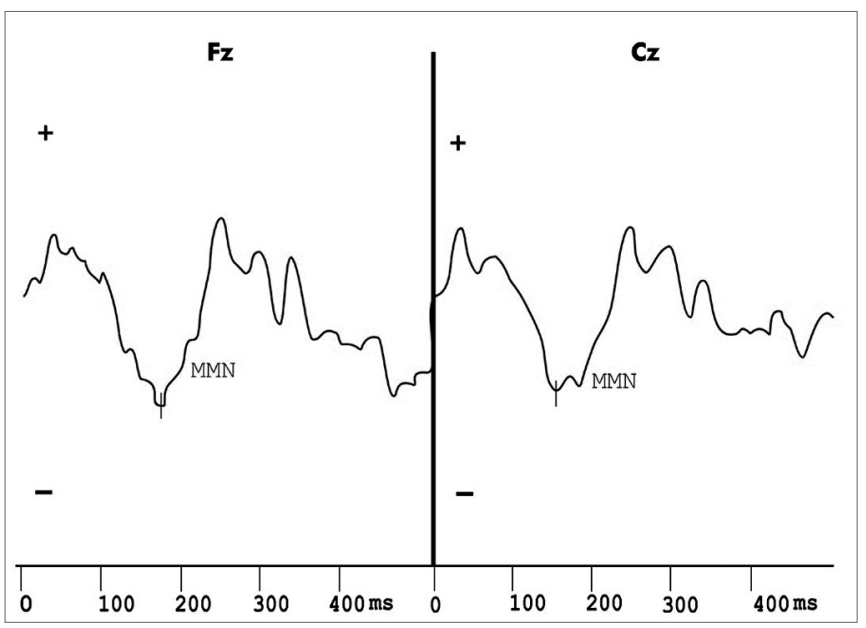

Figure 4. Mismatch Negativity shown by the difference wave obtained with frequent and infrequent stimuli in the hearing stimulation protocol with duration variation in the multiple sclerosis group.
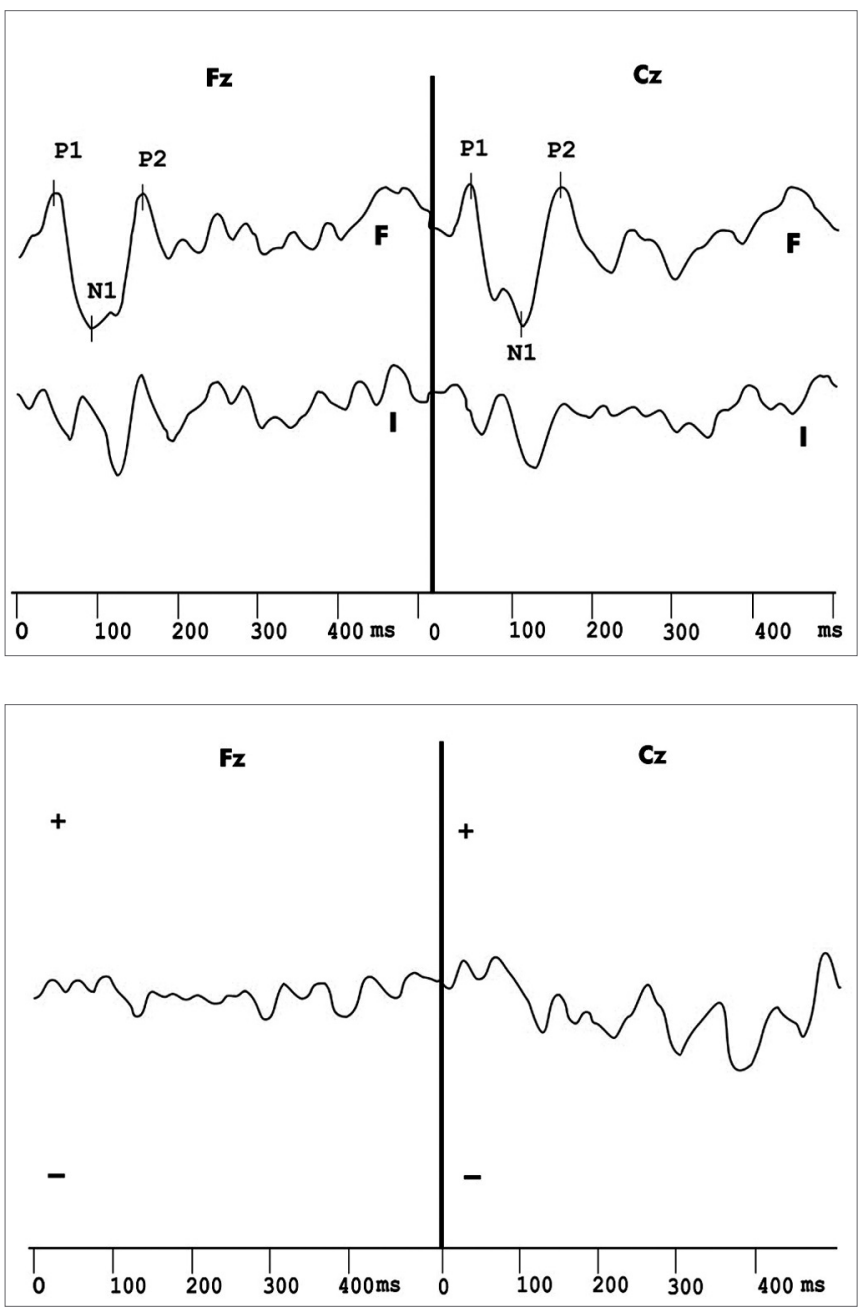

Figure 5. Exogenous long latency hearing evoked potentials recording and the difference wave obtained from frequent and infrequent stimuli, with no Mismatch Negativity in the duration variation protocol in the Multiple Sclerosis group. impairment assessed by PASAT in the MS group.

Figures 1 to 5 show wave potential examples of long latency and MMN in the control and multiple sclerosis groups.

\section{DISCUSSION}

MMN, described by Näätänen et $a .^{5}$, is a potential elicited by low probability hearing stimuli that represent a physical change in relation to a repetitive standard stimulation. It is automatically generated, regardless of the subject's attention, whenever an afferent stimulus does not coincide with the sensorial representation of the physical characteristics of the repetitive stimulation. The MMN generating process is of pre-attention characteristic, with neuro-electrical contributions of the frontal cortex, which participates in attention voluntary and involuntary mechanisms.

The major advantage brought about by the evoked potentials, when compared to other neurocognitive methods is that the former lets us record neuronal activities associated to brain processing, making it possible to assess cerebral areas that are activated during the processing of cognitive tasks. With the MMN it is also possible to estimate echoic memory duration; many studies submit the endogenous origin of such potential.

The goal of clinical studies that use event-related evoked potentials is to show either the presence or absence of responses that are different from those of normal subjects.

In order for one individual to recognize a complex acoustic response as similar to one previously known, the sensorial representation of such stimulus must activate afferent currents ${ }^{6}$. When the acoustic difference between standard tones and their deviant counterparts is small, it becomes easier for the subject to ignore the hearing stimuli. Näätänen 5 considers that deviations of up to $10 \%$ are efficient in producing a pure MMN. Lang et al. ${ }^{8}$ report that, with a great difference between stimuli, the neurons activated in the auditory cortex by deviant stimuli are not the same that are activated by the standard stimuli, and they recommend we should avoid large differences between stimuli. In the present study, the protocol with duration variation in stimuli caused proper stimuli difference in order to elicit MMN. Näätänen ${ }^{5}$ reports that when this difference exceeds the critical threshold, a P3a component may overlap the response, thus compromising MMN Recording. Sams et al. ${ }^{9}$ described a latency reduction and MMN amplitude increase with the increase in stimuli frequency difference. Böttcher-Gandor and Ullsperger10 used 1000 $\mathrm{Hz}$ Standard tones and $1500 \mathrm{~Hz}$ deviant tones, identifying MMN with its characteristic traces and amplitudes. Our stimuli protocol with frequency variation using the same frequencies was able to elicit MMN without P300 or its P3a subcomponent. 
The tone duration to be used in blocks in order to elicit MMN must be enough to establish the Standard stimuli neuronal representation. Paavilainen et al. ${ }^{11}$ reported that the stimuli duration may be small, at $50 \mathrm{~ms}$ or less if the intension is to register an MMN with frequency variation. They also recommend that the minimum standard and deviant stimulus duration were of $75 \mathrm{~ms}$ and $25 \mathrm{~ms}$ respectively, in the duration variation protocol, and $30 \mathrm{~ms}$ in the frequency variation protocol. These stimuli elicited MMN in both groups.

According to Schröger ${ }^{12}$, the stimuli intensity influence on the MMN creation, elicited by other alterations such as frequency or duration is yet to be determined. MMN can be recorded even at low stimuli intensities, when both the Standard and deviant stimuli can be perceived at $30 \mathrm{~dB}$ SPL. Escera and $\mathrm{Grau}^{7}$ proved MMN reproducibility using $85 \mathrm{~dB}$ SPL of stimulus intensity with supra-aural phones. In the present study, we chose to use stimuli both in the frequency variation protocol and the duration variation, at $55 \mathrm{~dB} \mathrm{HL}$, making it possible to Record the subjects in the control group and in the MS group.

Grau et al. ${ }^{7}$, using Standard stimuli of $75 \mathrm{~ms}$ in duration (likelihood $=0.83$ ) and $25 \mathrm{~ms}$ deviant stimuli (likelihood $=0.17$ ), with $700 \mathrm{~Hz}$ and $85 \mathrm{~dB}$ SPL of intensity, with binaural presentation and inter-stimuli intervals of $0.4 \mathrm{~s}$ and 4.0s in different stimulation blocks, observed the EPs in response to Standard stimuli with a typical morphology characterized by P1, N1 and P2 waves. These exogenous potentials are sensitive to the stimuli physical characteristics. MMN was seen in response to the two stimulation blocks, thus characterizing the endogenous nature of such potential. In the present study, the duration variation protocol, using $75 \mathrm{~ms}$ duration standard stimuli (likelihood = 0.80 ) and $25 \mathrm{~ms}$ deviant stimuli (likelihood $=0.20$ ), at 750 $\mathrm{Hz}$ and $55 \mathrm{~dB}$ HL of intensity, with binaural presentation and Constant maintenance of inter-stimuli intervals were able to elicit the P1, N1 and P2 exogenous potentials and the MMN in all control group subjects.

The MMN was present in 60\% of the MS individuals group in the hearing stimulation protocol with duration variation and in 45\% in the hearing stimulation protocol with frequency variation. In these recordings there was no statistically significant difference in MMN negative amplitude and latency when compared to the control group. Such findings confirm the echoic memory preservation in this group of MS individuals in whom the MMN was recorded, since they did not show statistically significant differences in negative amplitude and latencies when compared to the control group.

In MS, the electrophysiological evaluation of CNS functions carried out by visual evoked potentials, short latency auditory and somatosensitive potentials are well established. However, event-related EPs have been broadly studied because of their correlation with cognitive aspects, indicating integrity of the neurophysiological pathways, related to exogenous sensorial information. Endogenous evoked potentials, called event-related, are correlated to the cognitive function. The alterations found in these potentials are defined by wave extension or the lack of the characteristic peak formation.

The alterations found in the present study with MS patients were the lack of responses in $40 \%$ of the individuals in the hearing stimulation protocol with duration variation and in 55\% in the hearing stimulation protocol with frequency variation. Newton et al. ${ }^{14}$ found an incidence of $57 \%$ of cognitive potential alterations that included delays in wave latencies and lack of its characteristic morphology. Dijk et al. ${ }^{15}$ did not find differences in event-related evoked potentials in a group of 30 patients with MS, with an incidence of $15.6 \%$ of wave peak absence of all the potentials assessed.

Cognitive function reduction is a common manifestation in MS. The most frequent are memory, attention, processing speed and verbal fluency alterations. There may also be alterations in visual acuity and language. Some authors suggest that the MS cognitive alterations patterns are similar to those found in other diseases that affect these subcortical structures.

Neuropsychological tests make up the gold Standard in determining the cognitive function present in MS. Neuropsychologists have an array of tests to assess the most commonly affected functions.

The MSFC, developed by Cutter et al. ${ }^{20}$, based on an analysis of psychometric tests in MS patients is made up of three measures: walking skills, manual dexterity and cognitive function evaluation - by PASAT. Haase et al. ${ }^{22}$ developed a Brazilian version for the MSFC, made up of the following tests: 25 feet timed walk, 9 pin in the hole test and the PASAT. These authors concluded that processing speed is one of the PASAT functions and it is affected in MS.

The PASAT is considered a practical test, and one sensitive in recognizing MS cognitive impairment. The present study aimed at correlating MMN recording alterations with cognitive function impairment in a group of MS patients. The PASAT score average for males was of 27.8 and for females was of 28.43. Scores equal to or below the 25 th percentile were considered as cognitive impairment assessed by PASAT. Haase et al. ${ }^{22}$ defined this percentile for a control group with age, educational level and socioeconomical level matching those from the MS group.

We then used this study to compare MMN presence or absence with PASAT-measured cognitive impairment, making a qualitative data analysis. We found a statistically significant correlation between the lack of MMN wave with cognitive impairment.

Rao et $a .^{18}$ assessed the cognitive function in MS by means of an array of neuropsychologic tests, and 
found $25 \%$ of alterations in the attention assessment tests, measures by PASAT and the verbal fluency tests, without association with the reduction in cognitive function and the MS duration, depression, course of the disease or use of medication. In the present study, we found $57.5 \%$ of MS patients with PASAT alterations. Depression, MS and the use of medication were not considered, and none of the subjects were in MS exacerbation period. Giesser et al. ${ }^{16}$ found higher N100, P200 and P300 potential latencies in the MS patients with dementia; and worse neuropsychologic test performance related to an increase in P300 latency.

Gil et al. ${ }^{17}$ tried to correlate P300 latency increase with alterations in the neuropsychological tests in MS patients, and they did not find amplitude and latency differences in P200, N200 and P300 potentials among the subgroups classified by the McAlpine criteria. In the present study, it was possible to correlate MMN to alterations in a neuropsychological test.

Newton et al. ${ }^{14}$ reported that event-related evoked potential generation would be, in part, dependant on white matter integrity, and mild degrees of cognitive dysfunction would not always be detected by intellectual capacity assessment tests, thus the possibility of objective tests, carried out by event-related evoked potentials become a useful tool in clinical practice.

\section{CONCLUSIONS}

From this study using MMN in MS patients we may conclude that: when present in MS, MMN may be used as a supratemporal auditory cortex functional index, and its absence is related to cognitive impairment, found by the PASAT test.

Further studies are necessary in order to better understand MMN and its topographic distribution in the skull of MS patients, and thus allowing us to objectively assess attention and cognition, and better rehabilitate and follow these patients clinically.

\section{REFERENCES}

1. Chiappa KH. Principles of evoked potentials. In: Chiappa KH. Evoked Potentials in Clinical Medicine. Philadelphia: Lippincott -Raven Publishers; 1997. p.1-30.

2. Donchin E, Ritter W, McCallum WC. Cognitive psychophysiology: the endogenous components of the ERPs. In: Callaway AE, Tueting P, Koslow SH. Event - related potentials in man. New York: Academic Press; 1978. p.349-441.

3. Hillyard AS, Picton TW. Electrophysiology of cognition. In: Plum F. Handbook of Physiology. Maryland: Betmesda; 1987. p.519-84.

4. Escera C. Nuevas aplicaciones clínicas de los potenciales evocados cerebrales: Mismatch Negativity (MMN). Med Clin (Barc) 1997;108:7018.

5. Näätänen R, Gaillard AWK, Mäntysalo S. Early selective-attention effect on evoked potential reinterpreted. Acta Psychologica 1978;42:31329.

6. Näätänen R. The Mismatch Negativity: a powerful tool for cognitive neuroscience. Ear and Hearing 1995;16:6-18.
7. Grau C, Escera C, Yago E, Polo MD. Mismatch Negativity and auditory sensory memory evaluation: a new faster paradigm. Cognitive Neuroscience 1998;9:2451-6.

8. Lang AH, Eerola O, Korpilahti P, Holopainen I, Salo S, Aaltonen O. Practical issues in clinical application of Mismatch Negativity. Ear and Hearing 1995;16:118-30.

9. Sams M, Paavilainen P, Alho K, Näätänen R. Auditory frequency discrimination and event-related potentials. Eletroenceph Clin Neurophysiol 1985;62:437-48.

10. Böttcher-Gandor C, Ullsperger P. Mismatch Negativity in event-related potentials to auditory stimuli as a function of varying interstimulus interval. Psychophysiology 1992;29(5):546-50.

11. Paavilainen P, Jiang D, Lavikainen J, Näätänen R. Stimulus duration and sensory memory trace: an event-related potential study. Biological Psychology 1993;35:139-52.

12. Schröger E. Measurement and interpretation of the Mismatch Negativity. Behavior Research Methods, Instruments \& Computers 1998;30:131-45.

13. Poser CM, Paty DW, Scheinberg L, McDonald I, Davis FA, Ebers GC, Johnson KP, Sibley WA, Silberberg DH, Tourtellotte WW. New diagnostic criteria for multiple sclerosis: guidelines for research protocols. Annals of Neurology 1983;13(3):227-31.

14. Newton MR, Barret G, Callanan MM, Towell AD. Cognitive eventrelated potentials in multiple sclerosis. Brain 1989;112:1637-960.

15. Dijk JG, Jennekens-Schikel A, Caekebeke JFV, Singh A, Zwinderman $\mathrm{AH}$. What is the validity of an "abnormal" evoked or event-related potential in MS. The J Neurol Sci 1992;109:11-7.

16. Giesser BS, Schroeder MM, LaRocca NG, Kurtzberg D, Ritter W, Vaughan HG, Scheinberg LC. Endogenous event-related potentials in multiple sclerosis patients. Eletroenceph Clin Neurophysiol 1992;82:320-29.

17. Gil R, Zai L, Neau JP, Jonveaux T, Agbo C, Rosolacci, T, Burbaud $\mathrm{P}$, Ingrand P. Event-related auditory evoked potentials and multiple sclerosis. Eletroenceph Clin Neurophysiol 1992; 88:182-7.

18. Rao SM, Leo GJ, Bernardin L, Unverzagt F. Cognitive dysfunction in multiple sclerosis. Neurology 1991;41:685-91.

19. Fischer JS. Cognitive impairment in Multiple Sclerosis. In: Handbook of Multiple Sclerosis. New York: Marcel Dekker, Inc; 2001.p.23355

20. Cutter RG, Baier ML, Rudick RA, Cookfair DL, Fisher JS, Petkau J, Syndulko K, Weinshenker BG, Antel JP, Confavoreux C, Ellison GW, Lublin F, Miller AE, Rao SM, Reingold S, Thompson A, Willoughby E. Development of a multiple sclerosis functional composite as a clinical trial outcome measure. Brain 1999;122:871-82.

21. Gronwall DMA. Paced auditory addition task: a measure of recovery from concussion. Percep Motor Skills 1977;44:367-73.

22. Haase VG, Lima EP, Lacerda SS, Lana-Peixoto MA. Desenvolvimento da versão brasileira da multiple sclerosis functional composite measure (MSFC-BCTRIMS). Arq Neuropsiquiatr 2004;62(2-A):363-70 